\title{
Information Distance in Multiples
}

\author{
Paul M. B. Vitányi
}

\begin{abstract}
Information distance is a parameter-free similarity measure based on compression, used in pattern recognition, data mining, phylogeny, clustering and classification. The notion of information distance is extended from pairs to multiples (finite lists). We study maximal overlap, metricity, universality, minimal overlap, additivity and normalized information distance in multiples. We use the theoretical notion of Kolmogorov complexity which for practical purposes is approximated by the length of the compressed version of the file involved, using a real-world compression program.
\end{abstract}

Index Terms-Data mining, information distance, Kolmogorov complexity, multiples, pattern recognition, similarity.

\section{INTRODUCTION}

I $\mathrm{N}$ pattern recognition, learning and data mining one obtains information from objects containing information. This involves an objective definition of the information in a single object, the information to go from one object to another object in a pair of objects, the information to go from one object to any other object in a multiple of objects and the shared information between objects, [34].

The classical notion of Kolmogorov complexity [21] is an objective measure for the information in an a single object and information distance measures the information between a pair of objects [3]. This last notion has spawned research in the theoretical direction, among others [6], [30], [35], [37]-[39]. Research in the practical direction has focused on the normalized information distance, the similarity metric, which arises by normalizing the information distance in a proper manner and approximating the Kolmogorov complexity through real-world compressors [7]-[9], [26], This normalized information distance is a parameter-free, feature-free, and alignment-free similarity measure that has had great impact in applications. A variant of this compression distance has been tested on all time sequence databases used in the last decade in the major data mining conferences (sigkdd, sigmod, icdm, icde, ssdb, vldb, pkdd, pakdd) [18]. The conclusion is that the method is competitive with all 51 other methods used and superior in heterogenous data clustering and anomaly detection. In [4] it was shown that the method is resistant to noise. This theory has found many applications in pattern recognition, phylogeny,

Manuscript received May 20, 2009; revised November 15, 2010; accepted November 19, 2010. Date of current version March 16, 2011. This work was supported in part by the ESF QiT Programmme, the EU NoE PASCAL II and in part by the Netherlands BSIK/BRICKS project.

The author is with the National Research Center for Mathematics and Computer Science, The Netherlands (CWI), and also with the University of Amsterdam, CWI, Science Park 123, 1098XG Amsterdam, The Netherlands (e-mail: Paul.Vitanyi@cwi.nl).

Communicated by A. Krzyzak, Associate Editor for Pattern Recognition, Statistical Learning, and Inference.

Digital Object Identifier 10.1109/TIT.2011.2110130 clustering and classification. For objects that are represented as computer files such applications range from weather forecasting, software, earthquake prediction, music, literature, ocr, bioinformatics, to internet [1], [2], [5], [8]-[10], [12], [19], [20], [22], [23], [25], [31]-[33], [40]. For objects that are only represented by name, or objects that are abstract like "red," "Einstein," "three," the normalized information distance uses background information provided by Google, or any search engine that produces aggregate page counts. It discovers the "meaning" of words and phrases in the sense of producing a relative semantics. Applications run from ontology, semantics, tourism on the web, taxonomy, multilingual questions, to question-answer systems [13]-[15], [17], [36], [41]-[43]. For more references on either subject see the textbook [28] or Google Scholar for references to [8], [9], [26].

However, in many applications we are interested in shared information between many objects instead of just a pair of objects. For example, in customer reviews of gadgets, in blogs about public happenings, in newspaper articles about the same occurrence, we are interested in the most comprehensive one or the most specialized one. Thus, we want to extend the information distance measure from pairs to multiples.

\section{A. Related Work}

In [27] the notion is introduced of the information required to go from any object in a multiple of objects to any other object in the multiple. This is applied to extracting the essence from, for example, a finite list of internet news items, reviews of electronic cameras, tv's and so on, in a way that works better than other methods. Let $X$ denote a finite list of $m$ finite binary strings defined by $X=\left(x_{1}, \ldots, x_{m}\right)$, the constituting strings ordered length-increasing lexicographic. We use lists and not sets, since if $X$ is a set we cannot express simply the distance from a string to itself or between strings that are all equal. Let $U$ be the reference universal Turing machine, for convenience the prefix one as in Section II. We define the information distance in $X$ by $E_{\max }(X)=\min \left\{|p|: U\left(x_{i}, p, j\right)=x_{j}\right.$ for all $\left.x_{i}, x_{j} \in X\right\}$. It is shown in [27, Theorem 2] that

$$
E_{\max }(X)=\max _{x: x \in X} K(X \mid x)
$$

up to a logarithmic additive term. Define $E_{\min }(X)=$ $\min _{x: x \in X} K(X \mid x)$. In [27, Theorem 3], they state that for every list $X=\left(x_{1}, \ldots, x_{m}\right)$ we have

$$
E_{\min }(X) \leq E_{\max }(X) \leq \min _{i: 1 \leq i \leq m} \sum_{x_{i}, x_{k} \in X \& k \neq i} E_{\max }\left(x_{i}, x_{k}\right)
$$

up to a logarithmic additive term. This is not a corollary of (I.1) as stated in [27], but both inequalities follow from the definitions. The left-hand side (LHS) is interpreted as the program length of the "most comprehensive object that contains the most 
information about all the others [all elements of $X$ ]," and the right-hand side (RHS) is interpreted as the program length of the "most specialized object that is similar to all the others." The paper [27] develops the stated results and applications. It does not develop the theory in any detail. That is the purpose of the present paper.

\section{B. Results}

Information distance for multiples, that is, finite lists, appears both practically and theoretically promising. In nearly all cases below the results imply the corresponding ones for the pairwise information distance defined as follows. The information distance in [3] between strings $x_{1}$ and $x_{2}$ is $E_{\max }\left(x_{1}, x_{2}\right)=$ $\max \left\{K\left(x_{1} \mid x_{2}\right), K\left(x_{2} \mid x_{1}\right)\right\}$. In the current paper $E_{\max }(X)=$ $\max _{x: x \in X} K(X \mid x)$. These two definitions coincide for $|X|=2$ since $K(x, y \mid x)=K(y \mid x)$ up to an additive constant term. We investigate the maximal overlap of information (Theorem 3.1) which for $|X|=2$ specializes to Theorem 3.4 in [3], Corollary 3.2 shows (I.1) and Corollary 3.3 shows that the LHS of (I.2) can be taken to correspond to a single program embodying the "most comprehensive object that contains the most information about all the others" as stated but not argued or proved in [27]; metricity (Theorem 4.1) and universality (Theorem 5.2) which for $|X Y|=2$ (for metricity) and $|X|=2$ (for universality) specialize to Theorem 4.2 in [3]; additivity (Theorem 6.1); minimum overlap of information (Theorem 7.1) which for $|X|=2$ specializes to [29, Theorem 8.3.7] and the nonmetricity of normalized information distance for lists of more than two elements and certain proposals of the normalizing factor (Section VIII). In contrast, for lists of two elements we can normalize the information distance as in Lemma V.4 and Theorem V.7 of [26]. The definitions are of necessity new as are the proof ideas. Remarkably, the new notation and proofs for the general case are simpler than the mentioned existing proofs for the particular case of pairwise information distance.

\section{PRELIMINARIES}

Kolmogorov Complexity: This is the information in a single object [21]. The notion has been the subject of a plethora of papers. Informally, the Kolmogorov complexity of a finite binary string is the length of the shortest string from which the original can be losslessly reconstructed by an effective general-purpose computer such as a particular universal Turing machine. Hence it constitutes a lower bound on how far a lossless compression program can compress. For technical reasons we choose Turing machines with a separate read-only input tape, that is scanned from left to right without backing up, a separate work tape on which the computation takes place and a separate output tape. Upon halting, the initial segment $p$ of the input that has been scanned is called the input "program" and the contents of the output tape is called the "output." By construction, the set of halting programs is prefix free. We call $U$ the reference universal prefix Turing machine. This leads to the definition of "prefix Kolmogorov complexity" which we shall designate simply as "Kolmogorov complexity."

Formally, the conditional Kolmogorov complexity $K(x \mid y)$ is the length of the shortest input $z$ such that the reference universal prefix Turing machine $U$ on input $z$ with auxiliary information $y$ outputs $x$. The unconditional Kolmogorov complexity $K(x)$ is defined by $K(x \mid \epsilon)$ where $\epsilon$ is the empty string (of length 0 ). In these definitions both $x$ and $y$ can consist of a nonempty finite lists of finite binary strings. For more details and theorems that are used in the present work see Appendix I.

Lists: A list is a multiple $X=\left(x_{1}, \ldots, x_{m}\right)$ of $m<\infty$ finite binary strings in length-increasing lexicographic order. If $X$ is a list, then some or all of its elements may be equal. Thus, a list is not a set but an ordered bag of elements. With some abuse of the common set-membership notation we write $x_{i} \in X$ for every $i(0 \leq i \leq m)$ to mean that " $x_{i}$ is an element of list $X$." The conditional prefix Kolmogorov complexity $K(X \mid x)$ of a list $X$ given an element $x$ is the length of a shortest program $p$ for the reference universal Turing machine that with input $x$ outputs the list $X$. The prefix Kolmogorov complexity $K(X)$ of a list $X$ is defined by $K(X \mid \epsilon)$. One can also put lists in the conditional such as $K(x \mid X)$ or $K(X \mid Y)$. We will use the straightforward laws $K(\cdot \mid X, x)=K(\cdot \mid X)$ and $K(X \mid x)=K\left(X^{\prime} \mid x\right)$ up to an additive constant term, for $x \in X$ and $X^{\prime}$ equals the list $X$ with the element $x$ deleted.

Information Distance: To obtain the pairwise information distance in [3] we take $X=\left\{x_{1}, x_{2}\right\}$ in (I.1). Then(I.1) is equivalent to $E_{\max }\left(x_{1}, x_{2}\right)=\max \left\{K\left(x_{1} \mid x_{2}\right), K\left(x_{2} \mid x_{1}\right)\right\}$.

\section{MAXIMAL OVERLAP}

We use the notation and terminology of Section I-A. Define $k_{1}=E_{\min }(X), k_{2}=E_{\max }(X)$ and $l=k_{2}-k_{1}$. We prove a maximal overlap theorem: the information needed to go from any $x_{i}$ to any $x_{k}$ in $X$ can be divided in two parts: a single string of length $k_{1}$ and a string $r$ of length $l$ (possibly depending on $x_{i}$ ), everything up to an additive logarithmic term.

Theorem 3.1: A single program of length $k_{1}+K\left(m, k_{1}, k_{2}\right)+\log m+O(1)$ bits concatenated with a string of $l$ bits, possibly depending on $i$, suffice to find $X$ from $x_{i}$ for every $x_{i} \in X$. To find an arbitrary element $x_{k} \in X$ from $x_{i}$ it suffices to concatenate at most another $\log m$ bits, possibly depending on $i$ and $k$.

Proof: Enumerate the finite binary strings lexicographic length-increasing as $s_{1}, s_{2}, \ldots$.. Let $G=(V, E)$ be a graph defined as follows. Let $A$ be the set of finite binary strings and $B$ the set of vectors of strings in $A$ defined by $v=\left(s_{1}, \ldots, s_{m}\right)$ such that

$$
\begin{aligned}
\min _{j: 1 \leq j \leq m}\left\{K\left(s_{1}, \ldots, s_{m} \mid s_{j}\right)\right\} & \leq k_{1} \\
\max _{j: 1 \leq j \leq m}\left\{K\left(s_{1}, \ldots, s_{m} \mid s_{j}\right)\right\} & \leq k_{2} .
\end{aligned}
$$

Given $k_{1}$ and $k_{2}$ the set $B$ can be enumerated. Define $V=$ $A \bigcup B$. Define $E$ by length-increasing lexicographic enumerating $A \times B$ and put $(r s, v) \in E$ with $r s \in A$ and $v=$ $\left(s_{1}, \ldots, s_{m}\right) \in B$ if $s=s_{j}$ for some $j(1 \leq j \leq m)$, where $r$ is chosen as follows. It is the $\left\lceil i / 2^{k_{1}}\right\rceil$ th string of length $l$ where $i$ is the number of times we have used $s \in A$. So the first $2^{k_{1}}$ times we choose an edge $(\cdot s, \cdot)$ we use $0^{l}$, the next $2^{k_{1}}$ we use $0^{l-1} 1$ and so on. In this way, $i \leq 2^{k_{2}}$ so that $i / 2^{k_{1}} \leq 2^{l}$. By adding $r$ to $s$ we take care that the degree of $r s$ is at most $2^{k_{1}}$ and not at most $2^{k_{2}}$ as it could be without the prefix $r$. The degree of a node $v \in B$ is trivially $m$. 
In addition, we enumerate $B$ length-increasing lexicographic and "color" everyone of the $m$ edges incident with an enumerated vector $v \in B$ with the same binary string $c$ of length $k_{1}+\log m$. If $v=\left(s_{1}, \ldots, s_{m}\right)$ and $v$ is connected by edges to nodes $r_{1} s_{1}, \ldots, r_{m} s_{m}$, then choose $c$ as the minimum color not yet appearing on any edge incident with any $r_{j} s_{j}(1 \leq$ $j \leq m$ ). Since the degree of every node $r s \in A$ is bounded by $2^{k_{1}}$ and hence the colors already used for edges incident on nodes $r_{1} s_{1}, \ldots, r_{m} s_{m}$ number at most $\sum_{1 \leq j \leq m}\left(2^{k_{1}}-1\right)=$ $m 2^{k_{1}}-m$, a color is always available.

Knowing $m, k_{1}, k_{2}$ one can reconstruct $G$ and color its edges. Given an element $x$ from the list $X$ and knowing the appropriate string $r$ of length $l$ and the color $c$ of the edge $(r x, X)$, we can find $X$. Hence a single program, say $p$, of length $k_{1}+$ $K\left(m, k_{1}, k_{2}\right)+\log m+O(1)$ bits suffices to find $X$ from $r x$ for any $x \in X$ and with $|r|=l$. An additional $\log m$ bits suffice to select any element of $X$. Taking these $\log m$ bits so that they encode the difference from $i$ to $k \bmod m$ we can compute from every $x_{i} \in X$ to every $x_{k} \in X$ and vice versa with the same program $p$ of length $k_{1}+K\left(m, k_{1}, k_{2}\right)+\log m+O(1)$ concatenated with a string $r$ of length $l$ and a string of length $\log m$, both possibly depending on $i$ and $k$. Since we know $m, k_{1}, k_{2}$ from the fixed program $p$, where they are encoded as a self-delimiting prefix of length $K\left(m, k_{1}, k_{2}\right)$ say, we can concatenate these strings without separation markers and reconstruct them.

Corollary 3.2: Since $k_{1}+l=k_{2}$, the theorem implies (I.1), that is, [27, Theorem 2].

It is not a priori clear that $E_{\min }(X)$ in the LHS of (I.2) corresponds to a single program that represents the information overlap of every shortest program going from any $x_{i}$ to the list $X$. This seems in fact assumed in [27] where $E_{\min }(X)$ is interpreted as the [Kolmogorov complexity of] "the most comprehensive object that contains the most information about all the others." In fact, for every $x_{i} \in X$ we can choose a shortest program going from $x_{i}$ to the list $X$ so that these programs have pairwise no information overlap at all (Theorem 7.1). But here we have proved the following.

Corollary 3.3: The quantity $E_{\min }(X)$ corresponds to a single shortest program that represents the maximum overlap of information of all programs going from $x_{i}$ to the list $X$ for any $x_{i} \in X$.

\section{METRICITY}

We consider nonempty finite lists of finite binary strings, each list ordered length-increasing lexicographic. Let $\mathcal{X}$ be the set of such ordered nonempty finite lists of finite binary strings. A distance function $d$ on $\mathcal{X}$ is defined by $d: \mathcal{X} \rightarrow \mathcal{R}^{+}$where $\mathcal{R}^{+}$ is the set of nonnegative real numbers. Define $W=U V$ if $W$ is a list of the elements of the lists $U$ and $V$ and the elements of $W$ are ordered length-increasing lexicographical. A distance function $d$ is a metric if $X, Y, Z \neq \emptyset$ and

1) Positive definiteness: $d(X)=0$ if all elements of $X$ are equal and $d(X)>0$ otherwise.

2) Symmetry: $d(X)$ is invariant under all permutations of $X$.

3) Triangle inequality: $d(X Y) \leq d(X Z)+d(Z Y)$.
Theorem 4.1: The information distance for lists, $E_{\max }$, is a metric where the (in)equalities hold up to a $O(\log K)$ additive term. Here $K$ is the largest quantity involved in the metric (in)equalities.

Proof: It is clear that $E_{\max }(X)$ satisfies positive definiteness and symmetry up to an $O(\log K)$ additive term where $K=$ $K(X)$. It remains to show the triangle inequality.

Claim 4.2: Let $X, Y, Z$ be three nonempty finite lists of finite binary strings and $K=\max \{K(X), K(Y), K(Z)\}$. Then, $E_{\max }(X Y) \leq E_{\max }(X Z)+E_{\max }(Z Y)$ up to an $O(\log K)$ additive term.

Proof: By Theorem 3.1

$$
\begin{aligned}
& E_{\max }(X Y)=\max _{x: x \in X Y} K(X Y \mid x)=K\left(X Y \mid x_{X Y}\right) \\
& E_{\max }(X Z)=\max _{x: x \in X Z} K(X Z \mid x)=K\left(X Z \mid x_{X Z}\right) \\
& E_{\max }(Z Y)=\max _{x: x \in Z Y} K(Z Y \mid x)=K\left(Z Y \mid x_{Z Y}\right)
\end{aligned}
$$

equalities up to a $O(\log K)$ additive term. Here $x_{X Y}, x_{X Z}, x_{Z Y}$ are the elements for which the maximum is reached for the respective $E_{\max }$ 's.

Assume that $x_{X Y} \in X$, the case $x_{X Y} \in Y$ being symmetrical. Let $z$ be some element of $Z$. Then

$$
\begin{aligned}
K\left(X Y \mid x_{X Y}\right) & \leq K\left(X Y Z \mid x_{X Y}\right) \\
& \leq K\left(X Z \mid x_{X Y}\right)+K\left(Y \mid X Z, x_{X Y}\right) \\
& \leq K\left(X Z \mid x_{X Z}\right)+K(Y \mid X Z, z) \\
& \leq K\left(X Z \mid x_{X Z}\right)+K\left(Z Y \mid x_{Z Y}\right) .
\end{aligned}
$$

The first inequality follows from the general $K(u) \leq K(u, v)$, the second inequality by the obvious subadditive property of $K(\cdot)$, the third inequality since in the first term $x_{X Y} \in X Z$ and the $\max _{x: x \in X Z}\{K(X Z \mid x)\}$ is reached for $x=x_{X Z}$ and in the second term both $x_{X Y} \in X$ and for $z$ take any element from $Z$ and the fourth inequality follows by in the second term dropping $X$ from the conditional and moving $Z$ from the conditional to the main argument and observing that both $z \in Z Y$ and the $\max _{x: x \in Z Y}\{K(Z Y \mid x)\}$ is reached for $x=x_{Z Y}$. The theorem follows with (in)equalities up to an $O(\log K)$ additive term.

\section{UNIVERSALITY}

Let $X \in \mathcal{X}$. A priori we allow asymmetric distances. We would like to exclude degenerate distance measures such as $D(X)=1$ for all $X$. For each $d$, we want only finitely many lists $X$ such that $D(X) \leq d$. Exactly how fast we want the number of lists we admit to go to $\infty$ is not important; it is only a matter of scaling. For every distance $D$ we require the following density condition for every $x \in\{0,1\}^{*}$ :

$$
\sum_{X: x \in X \& D(X)>0} 2^{-D(X)} \leq 1 .
$$

Thus, for the density condition on $D$ we consider only lists $X$ with $|X| \geq 2$ and not all elements of $X$ are equal. Moreover, we consider only distances that are computable in some broad sense.

Definition 5.1: An admissible list distance $D(X)$ is a total, possibly asymmetric, function from $\mathcal{X}$ to the nonnegative real 
numbers that is 0 if all elements of $X$ are equal and greater than 0 otherwise (up to an additive $\log K$ additive term with $K=K(X)$ ), is upper semicomputable and satisfies the density requirement in (V.1).

Theorem 5.2: The list information distance $E_{\max }(X)$ is admissible and it is minimal in the sense that for every admissible list distance function $D(X)$ we have $E_{\max }(X) \leq D(X)$ up to an additive constant term.

Proof: It is straightforward that $E_{\max }(X)$ is a total realvalued function, is 0 only if all elements of $X$ are equal and unequal 0 otherwise (up to an $O(\log K)$ additive term with $K=K(X)$ ) and is upper semicomputable. We verify the density requirement of (V.1). For every $x \in\{0,1\}^{*}$, consider lists $X$ of at least two elements not all equal and $x \in X$. Define functions $f_{x}(X)=2^{-K(X \mid x)}$. Then, $f_{x}(X) \geq 2^{-E_{\max }(X)}$. It is easy to see that for every $x \in\{0,1\}^{*}$

$$
\begin{aligned}
X: x \in X \& E_{\max }(X)>0 & 2^{-E_{\max }(X)} \\
\leq & \sum_{X: x \in X \& E_{\max }(X)>0} f_{x}(X) \\
= & \sum_{X: x \in X \& E_{\max }(X)>0} 2^{-K(X \mid x)} \leq \sum 2^{-l(p)}
\end{aligned}
$$

where the RHS sum is taken over all programs $p$ for which the reference prefix machine $U$, given $x$, computes a finite list $X$ of at least two elements not all equal and such that $x \in X$. This sum is the probability that $U$, given $x$, computes such a list $X$ from a program $p$ generated bit by bit uniformly at random. Therefore, the RHS sum is at most 1 and $E_{\max }(X)$ satisfies the density requirement (V.1).

We prove minimality. Fix any $x \in\{0,1\}^{*}$. Since $D$ is upper semicomputable, the function $f$ defined by $f(X, x)=2^{-D(X)}$ for $X$ satisfying $x \in X$ and $D(X)>0$ and 0 otherwise, is lower semicomputable. Since $\sum_{X: x \in X \& D(X)>0} 2^{-D(X)} \leq 1$, we have $\sum_{X} f(X, x) \leq 1$ for every $x$. Note that given $D$ we can compute $f$ and hence $K(f) \leq K(D)+O(1)$. By the conditional version of Eq. (A.2) in the Appendix, that is [28, Theorem 4.3.2], we have $c_{D} \mathbf{m}(X \mid x \in X) \geq f(X, x)$ with $c_{D}=2^{K(f)}=2^{K(D)+O(1)}$, that is, $c_{D}$ is a positive constant depending on $D$ only. By the conditional version of Eq. (A.3) in the Appendix, that is [28, Theorem 4.3.4], we have for every $x \in X$ that $\log 1 / \mathbf{m}(X \mid x)=K(X \mid x)+O(1)$. Hence, for every $x \in X$ we have $\log 1 / f(X, x) \geq K(X \mid x)+\log 1 / c_{D}+O(1)$. Altogether, for every admissible distance $D$ and every $x \in$ $\{0,1\}^{*}$ and every list $X$ satisfying $x \in X$, there is a constant $c_{D}$ such that $D(X) \geq K(X \mid x)+\log 1 / c_{D}+O(1)$. Hence, $D(X) \geq E_{\max }(X)+\log 1 / c_{D}+O(1)$.

\section{ADDITIVITY}

Theorem 6.1: $E_{\max }$ is not subadditive: neither $E_{\max }(X)+$ $E_{\max }(Y) \leq E_{\max }(X Y)$ nor $E_{\max }(X)+E_{\max }(Y) \geq$ $E_{\max }(X Y)$, the (in)equalities up to logarithmic additive terms, holds for all lists $X, Y$.

Proof: Below, all (in)equalities are taken up to logarithmic additive terms. Let $x, y$ be strings of length $n$,
$X=(\epsilon, x)$ and $Y=(\epsilon, y)$ with $\epsilon$ denoting the empty word. Then $E_{\max }(X Y)=E_{\max }(\epsilon, \epsilon, x, y), E_{\max }(X)=K(x)$ and $E_{\max }(Y)=K(y)$. If $x=y$ and $K(x)=n$, then $E_{\max }(X Y)=E_{\max }(X)=E_{\max }(Y)=n$. Hence, $E_{\max }(X Y)<E_{\max }(X)+E_{\max }(Y)$.

Let $x, y$ be strings of length $n$ such that $K(x \mid y)=K(y \mid x)=$ $n, K(x), K(y) \geq n, X=(x, x)$ and $Y=(y, y)$. Then $E_{\max }(X Y)=E_{\max }(x, x, y, y)=\max \{K(x \mid y), K(y \mid x)\}=$ $n, E_{\max }(X)=0$ and $E_{\max }(Y)=0$. Hence, $E_{\max }(X Y)>$ $E_{\max }(X)+E_{\max }(Y)$.

Let $X=(x)$ and $Y=(y)$. Note that subadditivity holds for lists of singleton elements since $E_{\max }(x, y)=$ $\max \{K(x \mid y), K(y \mid x)\} \leq K(x)+K(y)$, where the equality holds up to an additive $O(\log \{K(x \mid y), K(y \mid x)\})$ term and the inequality holds up to an additive constant term.

\section{Minimal OVERLAP}

Let $X=\left(x_{1}, \ldots, x_{m}\right)$ and $p_{i}$ be a shortest program converting $x_{i}$ to $X(1 \leq i \leq m)$. Naively we expect that the shortest program that that maps $x_{i}$ to $X$ contains the information about $X$ that is lacking in $x_{i}$. However, this is too simple, because different short programs mapping $x_{i}$ to $X$ may have different properties.

For example, suppose $X=\{x, y\}$ and both elements are strings of length $n$ with $K(x \mid y), K(y \mid x) \geq n$. Let $p$ be a program that ignores the input and prints $x$. Let $q$ be a program such that $y \oplus q=x$ (that is, $q=x \oplus y$ ), where $\oplus$ denotes bitwise addition modulo 2 . Then, the programs $p$ and $q$ have nothing in common.

Now let $x$ and $y$ be arbitrary strings of length at most $n$. Muchnik [29], see also the textbook [28, Theorem 8.3.7], shows that there exists a shortest program $p$ that converts $y$ to $x$ (that is, $|p|=K(x \mid y)$ and $K(x \mid p, y)=O(\log n))$, such that $p$ is simple with respect to $x$ and therefore depends little on the origin $y$, that is, $K(p \mid x)=O(\log n)$. This is a fundamental coding property for individual strings that parallels related results about random variables known as the Slepian-Wolf and Csiszár-Körner-Marton theorems [11].

Theorem 7.1: Let $X=\left(x_{1}, \ldots, x_{m}\right)$ be a list of binary strings of length at most $n$. For every $x_{i} \in X$ there exists a string $p_{i}$ of length $K\left(X \mid x_{i}\right)$ such that $K\left(p_{i} \mid X\right)=O(\log m n)$ and $K\left(X \mid p_{i}, x_{i}\right)=O(\log m n)$.

Proof: Muchnik's theorem as stated before gives a code $p$ for $x$ when $y$ is known. There, we assumed that $x$ and $y$ have length at most $n$. The proof in [29] does not use any assumption about $y$. Hence we can extend the result to information distance in finite lists as follows. Suppose we encode the constituent list elements of $X$ self-delimitingly in altogether $m n+O(\log m n)$ bits (now $X$ takes the position of $x$ and we consider strings of length at most $m n+O(\log m n))$. Substitute $y$ by $x_{i}$ for some $i(1 \leq i \leq m)$. Then the theorem above follows straightforwardly from Muchnik's original theorem about two strings of length at most $n$.

The code $p_{i}$ is not uniquely determined. For example, let $X=$ $(x, y)$ and $z$ be a string such that $|x|=|y|=|z|=n, K(y \mid z)=$ $K(z \mid y) \geq n$ and $x=y \oplus z$. Then, both $z$ and $y \oplus z$ can be used 
for $p$ with $K(p \mid X)=O(1)$ and $K(X \mid p, y)=O(1)$. But $z$ and $y \oplus z$ have no mutual information at all.

Corollary 7.2: Let $X=\left(x_{1}, \ldots, x_{m}\right)$. For every string $x_{i}$ there is a program $p_{i}$ such that $U\left(p_{i}, x_{i}\right)=X(1 \leq i \leq m)$, where $\left|p_{i}\right|=K\left(X \mid x_{i}\right)$ and $K\left(p_{i}\right)-K\left(p_{i} \mid p_{j}\right)=K\left(p_{j}\right)-$ $K\left(p_{j} \mid p_{i}\right)=0(i \neq j)$ and the last four equalities hold up to an additive $O(\log K(X))$ term.

\section{NORMALIZED LIST INFORMATION DISTANCE}

The quantitative difference in a certain feature between many objects can be considered as an admissible distance, provided it is upper semicomputable and satisfies the density condition (V.1). Theorem 5.2 shows that $E_{\max }$ is universal in that among all admissible list distances in that always least. That is, it accounts for the dominant feature in which the elements of the given list are alike. Many admissible distances are absolute, but if we want to express similarity, then we are more interested in relative ones. For example, if two strings of 1000000 bits have information distance 1000 bits, then we are inclined to think that those strings are relatively similar. But if two strings of 1000 bits have information distance 1000 bits, then we find them very different.

Therefore, our objective is to normalize the universal information distance $E_{\max }$ to obtain a universal similarity distance. It should give a similarity with distance 0 when the objects in a list are maximally similar (that is, they are equal) and distance 1 when they are maximally dissimilar. Naturally, we desire the normalized version of the universal list information distance metric to be also a metric.

For pairs of objects, say $x, y$, the normalized version $e$ of $E_{\text {max }}$ defined by

$$
\begin{aligned}
e(x, y) & =\frac{E_{\max }(x, y)}{\max \{K(x), K(y)\}} \\
& =\frac{\max \{K(x, y \mid x), K(x, y \mid y)\}}{\max \{K(x), K(y)\}}
\end{aligned}
$$

takes values in $[0,1]$ and is a metric. Several alternatives for the normalizing factor $1 / \max \{K(x), K(y)\}$ do not work. Dividing by the length, either the sum or the maximum does not satisfy the triangle property. Dividing by $K(x, y)$ results in $e_{1}(x, y)=E_{\max }(x, y) / K(x, y)=\frac{1}{2}$ for $|x|=|y|=n$ and $K(x \mid y)=K(y \mid x) \geq n$ (and hence $K(x), K(y) \geq n$ ) and this is improper as $e_{1}(x, y)$ should be 1 in this case. We would like a proposal for a normalization factor for lists of more than two elements to reduce to that of (VIII.1) for lists restricted to two elements. This leads to the proposals below, which turn out to be improper.

As a counterexample to normalization take the following lists: $X=(x), Y=(y)$ and $Z=(y, y)$. With $|x|=|y|=n$ and the equalities below up to an $O(\log n)$ additive term we define: $K(x)=K(x \mid y)=K(x, y \mid y)=K(x, y, y \mid y)=n$, $K(y \mid x)=K(y, y \mid x)=K(x, y, y \mid x)=0.9 n$ and $K(y)=K(y, y)=0.9 n$. Using the symmetry of information (A.1) we have $K(x, y)=1.9 n$. Let $U, V, W$ be lists. We show that for the proposals below the triangle property $e(U V) \leq e(U W)+e(W V)$ is violated.
- Consider the normalized list information distance

$$
e(V)=\frac{K\left(V \mid x_{V}\right)}{K\left(V_{\max }\right)} .
$$

That is, we divide $E_{\max }(V)$ by $K\left(V_{\max }\right)$. Here, $K\left(V \mid x_{V}\right)=E_{\max }(V)$, with $K\left(V_{\max }\right)=\max _{i}\left\{K\left(V_{i}\right)\right\}$ where the list $V_{i}$ equals the list $V$ with the $i$ th element deleted $(1 \leq i \leq|V|)$. Then, with equalities holding up to $O((\log n) / n)$ we have: $e(X Y)=K(x, y \mid y) / K(x)=1, e(X Z)=$ $E_{\max }(X Z) / K\left((X Z)_{\max }\right)=K(x, y, y \mid y) / K(x, y)=\approx$ $\frac{1}{2}$ and $e(Z Y)=E_{\max }(Z Y) / K\left((Z Y)_{\max }\right)=$ $K(y, y, y \mid y) / K(y, y)=0$. Hence the triangle inequality does not hold.

- Instead of dividing by $K\left(V_{\max }\right)$ in (VIII.2) divide by $K\left(V^{\prime}\right)$ where $V^{\prime}$ equals $V$ with $x_{V}$ deleted. The same counterexample to the triangle inequality holds.

- Instead of dividing by $K\left(V_{\max }\right)$ in (VIII.2) divide by $K\left(\left\{V_{\max }\right\}\right)$ where $\left\{V_{\max }\right\}$ is the set of elements in $V_{\max }$. To equate the sets approximately with the corresponding lists, change $Z$ to $\left\{y_{1}, y_{2}\right\}$ where $y_{i}$ equals $y$ but with the $i$ th bit flipped $(i=1,2)$. Again, the triangle inequality does not hold.

- Instead of dividing by $K\left(V^{\prime}\right)$ in (VIII.2) divide by $K\left(\left\{V^{\prime}\right\}\right)$ where $\left\{V^{\prime}\right\}$ is the set of elements in $V^{\prime}$. Change $Z$ as in the previous item. Again, the triangle inequality does not hold.

\section{APPENDIX \\ KOLMOGOROV COMPLEXITY THEORY}

Theory and applications are given in the textbook [28]. Here we give some relations that are needed in the paper. The information about $x$ contained in $y$ is defined as $I(y: x)=$ $K(x)-K(x \mid y)$. A deep and very useful, result due to Levin and Kolmogorov [44] and for the prefix version [16] called symmetry of information shows that

$$
K(x, y)=K(x)+K(y \mid x)=K(y)+K(x \mid y)
$$

with the equalities holding up to $O(\log K)$ additive precision. Here, $K=\max \{K(x), K(y)\}$. Hence, up to an additive logarithmic term $I(x: y)=I(y: x)$ and we call this the mutual (algorithmic) information between $x$ and $y$.

The universal a priori probability of $x$ is $Q_{U}(x)=$ $\sum_{U(p)=x} 2^{-|p|}$. The following results are due to Levin [24].

There exists a lower semicomputable function $\mathbf{m}$ : $\{0,1\}^{*} \rightarrow[0,1]$ with $\sum_{x} \mathbf{m}(x) \leq 1$, such that for every lower semicomputable function $\bar{P}:\{0,1\}^{*} \rightarrow[0,1]$ with $\sum_{x} P(x) \leq 1$ we have

$$
2^{K(P)} \mathbf{m}(x) \geq P(x)
$$

for every $x$. Here $K(P)$ is the length of a shortest program for the reference universal prefix Turing machine to lower semicompute the function $P$. For every $x \in\{0,1\}^{*}$

$$
K(x)=-\log Q_{U}(x)=-\log \mathbf{m}(x)
$$

with equality up to an additive constant independent of $x$. Thus, the Kolmogorov complexity of a string $x$ coincides up to an ad- 
ditive constant term with the logarithm of $1 / Q_{U}(x)$ and also with the logarithm of $1 / \mathbf{m}(x)$. This result is called the "Coding Theorem" since it shows that the shortest upper semicomputable code is a Shannon-Fano code of the greatest lower semicomputable probability mass function.

\section{REFERENCES}

[1] C. Ané and M. Sanderson, "Missing the forest for the trees: Phylogenetic compression and its implications for inferring complex evolutionary histories," Systemat. Biol., vol. 54, no. 1, pp. 146-157, 2005.

[2] D. Benedetto, E. Caglioti, and V. Loreto, "Language trees and zipping," Phys. Rev. Lett., vol. 88, no. 4, p. 048702, 2002.

[3] C. H. Bennett, P. Gács, M. Li, P. M. B. Vitányi, and W. Zurek, "Information distance," IEEE Trans. Inf. Theory, vol. 44, pp. 1407-1423, 1998.

[4] M. Cebrián, M. Alfonseca, and A. Ortega, "The normalized compression distance is resistant to noise," IEEE Trans. Inf. Theory, vol. 53, pp. 1895-1900, 2007.

[5] X. Chen, B. Francia, M. Li, B. Mckinnon, and A. Seker, "Shared information and program plagiarism detection," IEEE Trans. Inf. Theory, vol. 50, pp. 1545-1550, 2004.

[6] A. V. Chernov, An. A. Muchnik, A. E. Romashchenko, A. K. Shen, and N. K. Vereshchagin, "Upper semi-lattice of binary strings with the relation $x$ is simple conditional to $y$," Theor. Comput. Sci., vol. 271, no. 1-2, pp. 69-95, 2002.

[7] R. Cilibrasi, P. M. B. Vitanyi, and R. de Wolf, "Algorithmic clustering of music based on string compression," Comput. Music J., vol. 28, no. 4, pp. 49-67, 2004.

[8] R. Cilibrasi and P. M. B. Vitanyi, "Clustering by compression," IEEE Trans. Inf. Theory, vol. 51, pp. 1523-1545, 2005.

[9] R. L. Cilibrasi and P. M. B. Vitanyi, "The Google similarity distance," IEEE Trans. Knowl. Data Eng., vol. 19, no. 3, pp. 370-383, 2007.

[10] C. C. Santos, J. Bernardes, P. M. B. Vitanyi, and L. Antunes, "Clustering fetal heart rate tracings by compression," in Proc. 19th IEEE Symp. Comput.-Based Med. Syst., 2006, pp. 685-690.

[11] T. M. Cover and J. A. Thomas, Elements of Information Theory. New York: Wiley, 1991.

[12] K. Emanuel, S. Ravela, E. Vivant, and C. Risi, "A combined statistical-deterministic approach to hurricane risk assessment," in Мапиscript Program in Athmospheres, Oceans and Climate. Cambridge, MA: MIT, 2005.

[13] D. Fensel and F. van Harmelen, "Unifying reasoning and search to web scale," IEEE Internet Comput., vol. 11, no. 2, pp. 94-96, 2007.

[14] G. Geleijnse and J. Korst, "Web-based artist categorization," in Proc. Conf. Music Inf. Retr., 2006, pp. 266-271.

[15] R. Gligorov, W. ten Kate, Z. Aleksovski, and F. van Harmelen, "Using Google distance to weight approximate ontology matches," in Proc. 16th Int. Conf. World Wide Web, NY, 2007, pp. 767-776.

[16] P. Gács, "On the symmetry of algorithmic information," Soviet Math. Dokl., vol. 15, pp. 1477-1480, 1974.

[17] B. Hu and B. Hu, "On capturing semantics in ontology mapping," World Wide Web, vol. 11, no. 3, pp. 361-385, 2008.

[18] E. Keogh, S. Lonardi, C. A. Ratanamahatana, L. Wei, H. S. Lee, and J. Handley, "Compression-based data mining of sequential data," Data Mining Knowl. Discov., vol. 14, no. 1, pp. 99-129, 2007.

[19] S. R. Kirk and S. Jenkins, "Information theory-based software metrics and obfuscation," J. Syst. Software, vol. 72, pp. 179-186, 2004.

[20] A. Kocsor, A. Kertész-Farkas, L. Kaján, and S. Pongor, "Application of compression-based distance measures to protein sequence classification: A methodology study," Bioinformatics, vol. 22, no. 4, pp. $407-412,2006$.

[21] A. N. Kolmogorov, "Three approaches to the quantitative definition of information," Problems Inf. Transmiss., vol. 1, no. 1, pp. 1-7, 1965.

[22] N. Krasnogor and D. A. Pelta, "Measuring the similarity of protein structures by means of the universal similarity metric," Bioinformatics, vol. 20, no. 7, pp. 1015-1021, 2004.

[23] A. Kraskov, H. Stögbauer, R. G. Andrzejak, and P. Grassberger, "Hierarchical clustering using mutual information," Europhys. Lett., vol. 70, no. 2, pp. 278-284, 2005.

[24] L. A. Levin, "Laws of information conservation (nongrowth) and aspects of the foundation of probability theory," Probl. Inf. Transm., vol. 10, pp. 206-210, 1974.

[25] M. Li, J. Badger, X. Chen, S. Kwong, P. Kearney, and H. Zhang, "An information-based sequence distance and its application to whole mitochondrial genome phylogeny," Bioinformatics, vol. 17, no. 2, pp. 149-154, 2001.
[26] M. Li, X. Chen, X. Li, B. Ma, and P. M. B. Vitányi, "The similarity metric," IEEE Trans. Inf. Theory, vol. 50, pp. 3250-3264, 2004.

[27] M. Li, C. Long, B. Ma, and X. Zhu, "Information shared by many objects," in Proc. 17th ACM Conf. Inf. Knowl. Manage., 2008, pp. $1213-1220$.

[28] M. Li and P. M. B. Vitányi, An Introduction to Kolmogorov Complexity and its Applications, 3rd ed. New York: Springer-Verlag, 2008.

[29] An. A. Muchnik, "Conditional complexity and codes," Theor. Comput. Sci., vol. 271, pp. 97-109, 2002.

[30] An. A. Muchnik and N. K. Vereshchagin, "Logical operations and Kolmogorov complexity II," in Proc. 16th IEEE Conf. Comput. Complex., 2001, pp. 256-265.

[31] M. Nykter, N. D. Price, M. Aldana, S. A. Ramsey, S. A. Kauffman, L. E. Hood, O. Yli-Harja, and I. Shmulevich, "Gene expression dynamics in the macrophage exhibit criticality," Proc. Nat. Acad. Sci. USA, vol. 105, no. 6, pp. 1897-1900, 2008.

[32] M. Nykter, N. D. Price, A. Larjo, T. Aho, S. A. Kauffman, O. Yli-Harja, and I. Shmulevich, "Critical networks exhibit maximal information diversity in structure-dynamics relationships," Phys. Rev. Lett., vol. 100, p. $058702(4), 2008$.

[33] H. Otu and K. Sayood, "A new sequence distance measure for phylogenetic tree construction," Bioinformatics, vol. 19, no. 6, pp. 2122-2130, 2003.

[34] P. Tan, V. Kumar, and J. Srivastava, "Selecting the right objective measure for association analysis," Inf. Syst., vol. 29, no. 4, pp. 293-313, 2004.

[35] A. K. Shen and N. K. Vereshchagin, "Logical operations and Kolmogorov complexity," Theor. Comput. Sci., vol. 271, no. 1-2, pp. $125-129,2002$.

[36] Z. Xiang, K. Wober, and D. R. Fesenmaier, "Representation of the online tourism domain in search engines," J. Travel Res., vol. 47, no. 2 , pp. 137-150, 2008.

[37] N. K. Vereshchagin and M. V. Vyugin, "Independent minimum length programs to translate between given strings," Theor. Comput. Sci., vol. 271, no. 1-2, pp. 131-143, 2002.

[38] M. V. Vyugin, "Information distance and conditional complexities," Theor. Comput. Sci., vol. 271, no. 1-2, pp. 145-150, 2002.

[39] M. V. Vyugin, "Systems of strings with high mutual complexity," Prob. Inform. Transmiss., vol. 39, no. 4, pp. 88-92, 2003.

[40] S. Wehner, "Analyzing worms and network traffic using compression," J. Comput. Secur., vol. 15, no. 3, pp. 303-320, 2007.

[41] W. Wong, W. Liu, and M. Bennamoun, "Featureless data clustering," in Handbook of Research on Text and Web Mining Technologies. New York: Idea Group Inc., 2008, ch. IX, pp. 141-164.

[42] X. Zhang, Y. Hao, X. Zhu, and M. Li, "Information distance from a question to an answer," in Proc. 13th ACM SIGKDD Int. Conf. Knowledge Discovery and Data Mining, 2007, pp. 874-883.

[43] J. Zhou, S. Wang, and C. Cao, "A Google-based statistical acquisition model of Chinese lexical concepts," in Proc. 2nd Conf. Knowl. Sci., Eng. Manage., Lect. Notes Comp. Sci., 2007, vol. 4798, pp. 243-254.

[44] A. K. Zvonkin and L. A. Levin, "The complexity of finite objects and the development of the concepts of information and randomness by means of the theory of algorithms," Russian Math. Surveys, vol. 25, no. 6 , pp. $83-124,1970$.

Paul M. B. Vitányi received the Ph.D. degree from the Free University of Amsterdam in 1978.

He is a CWI Fellow with the National Research Institute for Mathematics and Computer Science, Netherlands, CWI, and Professor of Computer Science with the University of Amsterdam. He has worked on cellular automata, computational complexity, distributed and parallel computing, machine learning and prediction, physics of computation, Kolmogorov complexity, information theory, quantum computing, publishing about 200 research papers and some books. Together with $\mathrm{M}$. Li, they pioneered applications of Kolmogorov complexity and coauthored An Introduction to Kolmogorov Complexity and its Applications (New York: Springer-Verlag, 1993), (3rd ed. 2008) parts of which have been translated into Chinese, Russian, and Japanese. Web page: http://www.cwi.nl/ $\sim$ paulv/

Dr. Vitányi serves on the editorial boards of Distributed Computing (1987-2003), Information Processing Letters, Theory of Computing Systems, Parallel Processing Letters, International Journal of Foundations of Computer Science, Entropy, Information, Journal of Computer and Systems Sciences (guest editor), and elsewhere. He received a Knighthood (Ridder in de Orde van de Nederlandse Leeuw) in 2007. 